\title{
Smart Cyclodextrin-Based Drug Delivery Systems: Stimuli-Responsiveness and Controlled Release
}

\author{
Shohreh Peimanfard ${ }^{1,2}$, Fabrizio Caldera*2, Alberto Rubin Pedrazzo ${ }^{2}$ and Nilesh Kumar Dhakar ${ }^{2}$ \\ ${ }^{1}$ Department of Biotechnology, University of Isfahan, Iran \\ ${ }^{2}$ Department of Chemistry, University of Torino, Italy
}

Received: 制: September 27, 2018; Published: 眥: October 09, 2018

*Corresponding author: Fabrizio Caldera, Department of Chemistry, University of Torino, Torino, Italy

\section{Mini Review}

Cyclodextrins (CDs), a family of macrocyclic oligosaccharides, are well known as drug carriers due to their specific structures and properties. Their adequate size and truncated cone-shaped architecture with inner slightly hydrophobic cavity and outer hydrophilic surface provide suitable space for interaction with many types of organic and inorganic compounds, including ions, proteins and oligonucleotides. The most common members of this family, $\alpha-C D, \beta-C D$ and $\gamma$-CD, which are termed parent CDs, possess 6, 7 and $8 \alpha$-D-glucopyranose units respectively. Among them, $\beta$-CD is able to form inclusion complexes with most of drugs, thanks to its medium-size inner cavity. As a result of their attributes in improving solubility, stability and bioavailability of guest molecules, CDs are widely used in pharmaceutical applications. All these useful properties can be further enhanced by employing water soluble derivatives of CDs, such as hydroxypropyl and sulfobutyl ether CDs. Benefiting from self-assembly and supramolecular host-guest interactions, CD-based inclusion complexes can result in different nanoarchitectures which endow improved characteristics as higher loading capacity, targeted and controlled release.

Through developing stimuli-responsive and controlled drug delivery systems (DDSs), dosage and frequency of drug administration will be reduced, thus leading to less toxicity, less side effects and higher efficacy. Since supramolecular non-covalent interactions between CDs and guest molecules may possess good stability and capability of response to many stimuli triggers, smart CD-based DDSs have been developed and attract lots of attention [1-10]. In this mini review, we will focus on the stimuli-responsive properties of CD-based DDSs. These specifically engineered nanoscaled delivery systems can be considered to be promising smart nanocarriers for controlled and targeted drug delivery. The release of drug from stimuli-responsive CD-based DDSs can be triggered by internal or external stimulation. In the case of cancer, as well as other diseases, alterations of the intracecellular environment are observed in abnormal tissues. Such differences in $\mathrm{pH}$, redox conditions and enzyme type and value can play the role of internal triggers. Many types of CD-based DDSs have been specifically designed to exploit internal stimuli as triggering factors [10-15].

Applying external stimuli offers the advantage of more precise control at the disease location and administration times. But if the external trigger is a chemical compound which should be provided to the cells, then it may accumulate in the body and cause side effects or toxicity $[10,16]$. CD-based DDSs can be categorized in two groups, depending on the role played by the guest molecules. In the first category, the DDS nanostructure is formed by the inclusion complex self-assembling between stimuli-sensitive guest molecules and the internal cavities of CDs. Therefore, drug molecules can be accommodated in the interstitial voids amidst CDs but not in the interior cavities. Stimuli-induced changes in the chemical structure of the guest molecules will result in the dissociation of the inclusion complex, disruption of the DDS structure and subsequent release of the loaded drug. Ferrocene, azobenzene and benzimidazole are three of the most common guests which can respond to redox, light and $\mathrm{pH} / \mathrm{CO} 2$ stimuli, respectively. Cheng et al. fabricated $\mathrm{pH}-$ triggered CD-based DDSs through host-guest interaction between thiolated $\beta$-CD and ferrocene derivative.

The so obtained supramolecular nanoparticles could entrap Doxorubicin (Dox) in their cores efficiently. In low pH environments nanoparticles disassembled and Dox was released rapidly. Considering their good stability, drug loading/release ability and tunable properties of nanostructure assembly/disassembly, this $\mathrm{pH}$ triggered CD-based DDS can be applied to target acidic cancer cells [17]. Previous studies on this category of stimuliresponsive DDSs have been reviewed elsewhere [10]. In the other category, drugs are mostly hosted in the internal cavities of CDs as in usual CD-based DDSs and they can be released by internal or external triggers. Stimuli-responsive linkers can be incorporated into the nanostructure via chemical reactions. By introducing 2-hydroxyethyl disulfide in the reaction solution, Trotta et al. have fabricated CD-based nanosponges with disulfide bridges which are 
cleavable in the presence of intracellular GSH. Cancer cells possess a higher amount of GSH, in comparison with normal cells, which causes reduction of disulfide bonds and then further drug release inside cancer cells.

Considering the higher level of GSH in chemo-resistant tumor cells, GSH has been indicated as an excellent trigger for controlled and targeted drug release in cancer therapy. Dox was loaded in the nanosponges and subsequently released as a function of GSH concentration. GSH-responsive nanosponges exhibited greater anti-proliferation effect on tumor cells with higher GSH content [18]. In a complementary in vitro and in vivo study, Daga et al, reported high effectiveness of mentioned GSH-responsive nanosponge in reducing mice tumor growth and decreasing number of proliferating Ki67 positive cells in comparison with free Dox. In light of the increased drug bioavailability, controlled release and considerable anti-proliferating and anti-tumor effect, GSHresponsive nanosponges can be considered a promising stimuliresponsive CD-based DDS for cancer treatment [19]. In another study, macroscopic mechanical force has been used as a trigger for releasing drug from a CD-based hydrogel. Ondansetron was released by mild mechanical compression, mimicking operation by a patient's hand. This external stress can change the inclusion complex conformation or stability, which can result in disassembly and release of the drug. This novel mechanical triggered CD-based DDS provides on-demand and in-situ administration, which could also be exploited for developing novel smart DDSs for anticancer drugs and insulin [20].

\section{Conclusion}

In recent years, CDs have been of great interest as excellent candidates for fabricating versatile nano DDSs due to their commercial availability, easy functionalization, low immunogenicity, biocompatibility and safety. Currently, parent CDs have been used to design and fabricate drug nanocarriers suitable for several administration routes including dermal, buccal, oral, sublingual, rectal, ophthalmic, intramuscular, and intravenous. These CD-based inclusion complexes, however, are not able to provide ideal controlled and targeted drug delivery. But the possibility of reversible inclusion complex formation between CDs and various guest molecules, in association with versatile exclusive properties of CDs, offer a route towards the fabrication of advanced supramolecular structures with enormous potential for an extensive range of applications.

In spite of the fact that diverse successful studies have been carried out for CD-based DDSs, still there is not a comprehensive approach to overcome all important drawbacks including systemic biocompatibility, supreme pharmacokinetics and pharmacodynamics, as well as controlled and targeted drug delivery. The fabrication of smart CD-based DDSs, capable of responding to external or internal triggers, such as temperature, $\mathrm{pH}$, light, and redox variations, put forward a solution to achieve extracellular stability and effective intracellular drug release. Yet, there is often a long way to go from promising theoretical and experimental results to an efficacious clinical therapy. In spite of these challenges and with regard to the notable clinical success of parent CDs, their derivatives, as well as novel CD-based nanoarchitectures, the future of smart CD-based DDSs is promising. In conclusion, the numerous investigations in this area pave the way to the next generation of smart nanotherapeutics, towards the enhanced controlled and targeted drug delivery.

\section{References}

1. Uekama K, Hirayama F, Irie T (1998) Cyclodextrin drug carrier systems. Chem Rev 98(5): 2045-2076.

2. Singh M, Sharma R, Banerjee UC (2002) Biotechnological applications of cyclodextrins. Biotechnol Adv 20(5-6): 341-359.

3. Davis ME, Brewster ME (2004) Cyclodextrin-based pharmaceutics: past, present, and future. Nat Rev Drug Discov 3(12): 1023-1035.

4. Saenger W, Jacob J, Gessler K, Steiner T, Hoffmann D, et al. (1998) Structures of the Common Cyclodextrins and Their Larger AnaloguesBeyond the Doughnut. Chem Rev 98(5): 1787-1802.

5. Zhang JX, Ma PX (2013) Cyclodextrin-based supramolecular systems for drug delivery: recent progress and future perspective. Adv Drug Delivery Rev 65(9): 1215-1233.

6. Wenz G (1994) Cyclodextrins as Building Blocks for Supramolecular Structures and Functional Units. Angew Chem Int Ed Engl 33: 803-822.

7. Irie T, Uekama K (1997) Pharmaceutical applications of cyclodextrins. III. Toxicological issues and safety evaluation. J Pharm Sci 86: 147-162.

8. Hirayama F, Uekama K (1999) Cyclodextrin-based controlled drug release system. Adv Drug Deliv Rev 36(1): 125-141.

9. Loftsson T, Brewster ME (2011) Pharmaceutical applications of cyclodextrins: effects on drug permeation through biological membranes. J Pharm Pharmacol 63(9): 1119-1135.

10. Peng L, Liu S, Feng A, Yuan J (2017) Polymeric Nanocarriers Based on Cyclodextrins for Drug Delivery: Host-Guest Interaction as StimuliResponsive Linker. Mol Pharmaceutics 14(8): 2475-2486.

11. Kikuzawa A, Kida T, Akashi M (2007) Synthesis of Stimuli-Responsive Cyclodextrin Derivatives and Their Inclusion Ability Control by Ring Opening and Closing Reactions. Organic Letters 9(20): 3909-3912.

12. Dan Z, Cao H, He X, Zeng L, Zou L, et al. (2015) Biological stimuliresponsive cyclodextrin-based host-guest nanosystems for cancer therapy. International Journal of Pharmaceutics 483(1-2): 63-68.

13. Dai Y, Zhang X (2018) Dual stimuli-responsive supramolecular polymeric nanoparticles based on poly( $\alpha$-cyclodextrin) and acetal-modified $\beta$-cyclodextrin-azobenzene. J Polym Res 25: 102.

14. Zhu CL, Wang XW, Lin ZZ, Xie ZH, Wang XR (2014) Cell microenvironment stimuli-responsive controlled-release delivery systems based on mesoporous silica nanoparticles, Journal of Food and Drug Analysis 22(1): 18-28.

15. Bai L, Zhao Q, Wang J, Gao Y, Sha Z, et al. (2015) Mechanism study on pH-responsive cyclodextrin capped mesoporous silica: effect of different stalk densities and the type of cyclodextrin. Nanotechnology 26(16): 165704-165715.

16. Ding C, Tong L, Feng J, Fu J (2016) Recent Advances in Stimuli-Responsive Release Function Drug Delivery Systems for Tumor Treatment. Molecules 21(12): 1715.

17. Cheng JG, Zhang YM, Liu Y (2018) Supramolecular Assembly of Thiolated Cyclodextrin and Ferrocene Derivative for Controlled Drug Delivery. Chem Nano Mat 4: 758-763.

18. Trotta F, Caldera F, Dianzani C, Argenziano M, Barrera G, et al. (2016) Glutathione Bioresponsive Cyclodextrin Nanosponges. Chem Plus Chem 81: $439-443$. 
19. Dago M, Ulllio C, Argenziano M, Dianzani C, Cavalli R, et al. (2016) GSH targeted nanosponges increase doxorubicin induced toxicity "in vitro" and "in vivo" in cancer cells with high antioxidant defenses. Free Radical Biology and Medicine 97: 24-37.

ISSN: 2574-1241

DOI: 10.26717/BJSTR.2018.09.001850

Fabrizio Caldera. Biomed J Sci \& Tech Res

(C) This work is licensed under Creative

Submission Link: https://biomedres.us/submit-manuscript.php
20. Izawa H, Kawakami K, Sumita M, Tateyama Y, Hillab JP, et al. (2013) $\beta$-Cyclodextrin-crosslinked alginate gel for patient-controlled drug delivery systems: regulation of host-guest interactions with mechanical stimuli. J Mater Chem B1: 2155-2161.

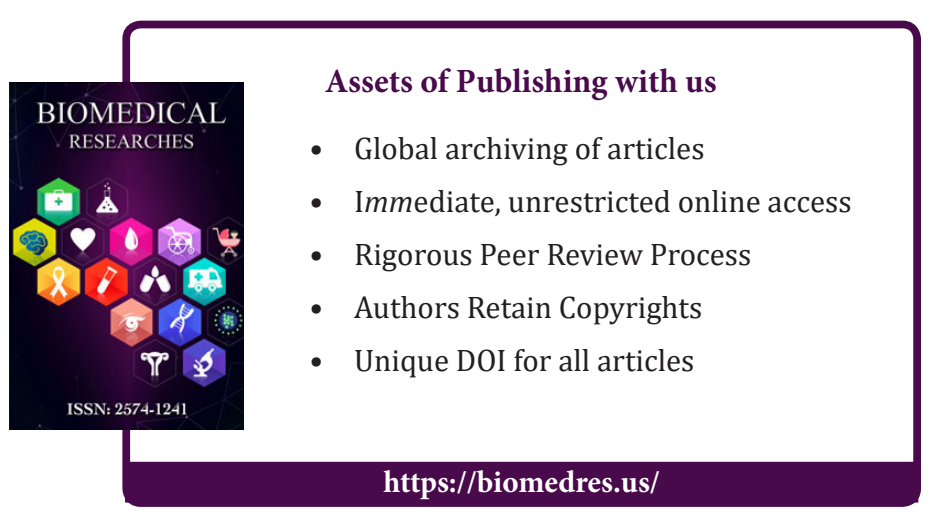

